\title{
Aplicação do Aprendizado de Máquina para Previsão do Tempo de Espera dos Serviços Públicos Federais Brasileiros
}

\author{
Vítor G. de Menezes ${ }^{1}$, Glauco V. Pedrosa ${ }^{1,2}$, Michel A. Ribeiro ${ }^{2}$, \\ Rejane M. da C. Figueiredo ${ }^{1,2}$ \\ ${ }^{1}$ Faculdade do Gama - Universidade de Brasilia (UnB) \\ Brasilia - DF - Brasil \\ ${ }^{2}$ Programa de Pós-Graduação em Computação Aplicada - Universidade de Brasilia (UnB) \\ Brasilia - DF - Brasil \\ $\{$ glauco.pedrosa, rejanecosta\}@unb.br
}

\begin{abstract}
This paper uses machine learning techniques to predict public service waiting times. The waiting time analyzed in this work refers to the period (in days) of the duration from the service request to the actual delivery to the requesting user. This work was developed in cooperation with the Brazilian Government, which conducted an exploratory interview with managers of 289 federal services. Then, a data mining was carried out to identify a set of variables that would allow to predict with high effectiveness the waiting time of a service from its managerial aspects. Two models are presented in this article: a predictive model for services offered to individuals, with an accuracy of $77 \%$ and another for companies with an accuracy of $70 \%$.
\end{abstract}

Resumo. Este artigo utiliza técnicas de aprendizado de máquina para prever o tempo de espera dos serviços públicos. O tempo de espera analisado neste trabalho se refere ao período (em dias) da duração desde a solicitação do serviço até a efetiva entrega ao usuário solicitante. Este trabalho foi desenvolvido em cooperação com o Governo Brasileiro, que realizou uma entrevista exploratória com gestores de 289 serviços federais. Em seguida, foi realizada uma mineração de dados para identificar um conjunto de variáveis que permitissem prever com alta efetividade o tempo de espera de um serviço a partir de seus aspectos gerenciais. Dois modelos são apresentados neste artigo: um modelo preditivo para serviços oferecidos a pessoas físicas, com acurácia de $77 \%$ e outro para pessoas jurídicas com acurácia de $70 \%$.

\section{Introdução}

Uma parte importante do processo de melhoria na prestação de serviços públicos aos cidadãos é a avaliação da qualidade dos serviços prestados [Alanezi et al. 2011]. Ao se avaliar a qualidade de um serviço, o tempo de espera é uma das principais causas de insatisfação e irritação [Soares et al. 2019, Pareja et al. 2016, Bielen and Demoulin 2007]. Por isso, é importante para uma organização identificar possíveis fatores que possam gerar atrasos e, assim, promover esforços para garantir uma melhoria no serviço prestado. No âmbito governamental não é diferente: o cidadão espera rapidez na obtenção do serviço prestado pelo órgão publico. Por isso, os gestores públicos precisam aplicar esforços para tornar os processos mais eficientes e diminuir o tempo 
de espera para atendimento, aumentando também a satisfação do usuário com o serviço recebido.

Nos últimos anos, a Secretaria de Governo Digital do Ministério da Economia vêm realizando pesquisas de gestão da qualidade nas instituições da Administração direta e indireta do governo federal brasileiro. Tal pesquisa possibilitou a identificação de algumas características na gestão de alguns serviços públicos, tais como: a visão da alta direção sobre a coerência entre a missão da instituição e o que é ofertado pelo serviço, os canais de interação e reclamação, quantidade de áreas existentes na prestação do serviço, medição do tempo de espera, entre outros aspectos gerenciais.

A crescente adoção pelos governos de ferramentas para analisar sua vasta quantidade de dados a fim de compreendê-los nesse novo contexto social abre oportunidade para a utilização de técnicas de Inteligência Artificial e o desenvolvimento de serviços orientados cognitivamente [Manocha et al. 2018]. De fato, o uso de sistemas para mineração de dados está cada vez mais sendo usado para envolver a inteligência artificial nos processos de tomada de decisão e abraçar a inovação na prestação de serviços públicos [Arias and Maçada 2018].

O objetivo deste trabalho é apresentar um modelo preditivo do tempo de espera dos serviços públicos usando os dados de gestão coletados pela Secretaria de Governo Digital do Ministério da Economia. Busca-se com este trabalho, identificar quais são as variáveis de gestão mais importantes que podem influenciar no tempo de espera de um serviço público. Assim, com a identificação dessas variáveis é possível planejar estratégias assertivas de intervenção e de alta efetividade, contribuindo para melhorar a qualidade do serviço prestado.

Este artigo apresenta, na seção 2, uma breve descrição sobre o instrumental de avaliação da gestão da qualidade dos serviços públicos brasileiros desenvolvido pela secretaria de Governo Digital do Ministério da Economia. Em seguida, na seção 3 são apresentados os materiais e métodos utilizados para a mineração dos dados e construção dos modelos preditivos. Na seção 4, algumas discussões sobre os modelos preditivos são apresentadas juntamente com a acurácia dos modelos usando diferentes técnicas de classificação supervisionada. $\mathrm{O}$ artigo conclui com um resumo destacando as principais contribuições do trabalho.

\section{Avaliação da Gestão dos Serviços Públicos Brasileiros}

Essa seção tem o objetivo de apresentar o instrumento desenvolvido pela Secretaria de Governo Digital do Ministério da Economia para avaliar a qualidade de gestão dos serviços públicos brasileiros. Esse instrumento é um questionário cujas perguntas envolvem 5 (cinco) dimensões gerenciais propostas pelo modelo Simplificando Vidas do BID (Banco Interamericano de Desenvolvimento) [Pareja et al. 2016]:

- Gestão de Processo

- Recursos

- Tecnologia da Informação

- Regulação e Gestão

- Relações com os cidadãos

As questões associadas a cada dimensão listada acima, assim como o padrão de respostas, foram definidas através de uma oficina realizada entre os dias 03 e 12 
de setembro de 2018 com um grupo de gestores de serviços públicos de 9 instituições federais, totalizando $5 \%$ do número de respostas esperadas para a pesquisa definitiva. A partir dessa oficina, um questionário preliminar foi elaborado e, posteriormente aprimorado, já que foram recebidos 68 comentários e sugestões de melhoria. Do total de sugestões, $88,88 \%$ foram incorporadas na versão final.

A Tabela 1 mostra o questionário de teste validado após o teste inicial. O questionário final apresenta 54 indicadores (questões). Foi solicitado a cada gestor de um serviço público federal que respondesse objetivamente à cada uma das questões apresentadas neste questionário. Algumas perguntas permitem apenas respostas binárias (sim-não) e outras seguiam uma escala Likert-4, na qual: 0 - Discordo totalmente; 1 Discordo; 3 - concordo; e 4 - Concordo plenamente. Apenas 3 perguntas ( $\mathrm{n}^{o} 3,4$ e 5) são questões numéricas de resposta aberta.

A questão $\mathrm{n}^{o} 2$ do questionário da Tabela 1 sobre o tempo de espera, se refere ao período (em dias) até a efetiva entrega do serviço ao usuário desde a sua solicitação. Para essa pergunta, 3 níveis foram estabelecidos:

- Tempo de Espera Baixo: até 29 dias

- Tempo de Espera Regular: de 30 a 60 dias

- Tempo de Espera Alto: acima de 60 dias

Tabela 1. Questionário respondido pelos gerentes de serviços públicos brasileiros

\begin{tabular}{|l|l|l|}
\hline ID & Pergunta & Resposta \\
\hline Q1 & $\begin{array}{l}\text { Existem medidas do tempo de espera do usuário até a entrega do } \\
\text { serviço? (Entrega: ato administrativo final na prestação do serviço) }\end{array}$ & Sim/Não \\
\hline Q2 & Quantos dias o usuário espera até que o serviço seja entregue? & Nível(1,2,3) \\
\hline Q3 & $\begin{array}{l}\text { Quantas áreas da instituição estão envolvidas na prestação do serviço? } \\
\text { (Área: setor que representa o nível hierárquico mais baixo do } \\
\text { organograma da instituição) }\end{array}$ & Aberta \\
\hline Q4 & $\begin{array}{l}\text { Em média, com que frequência o usuário interage com a instituição até } \\
\text { a entrega do serviço? (Interação: toda vez que um usuário interage com } \\
\text { a instituição durante a prestação do serviço) }\end{array}$ & Aberta \\
\hline Q5 & Quantos documentos o usuário precisa apresentar ao serviço? & Aberta \\
\hline & Como os usuários podem registrar sua reclamação ou sugestão? & Sim/Não \\
\hline Q6 & Ouvidoria da Instituição & Sim/Não \\
\hline Q7 & Pessoalmente & Sim/Não \\
\hline Q8 & Telefone & Sim/Não \\
\hline Q9 & Website & Sim/Não \\
\hline Q10 & Portal da Instituição & Sim/Não \\
\hline Q11 & e-Ouv & Sim/Não \\
\hline Q12 & Chat ou Chatbot & Sim/Não \\
\hline Q13 & APP & Sim/Não \\
\hline Q14 & Formulário em Papel & Sim/Não \\
\hline Q15 & $\begin{array}{l}\text { Sua instituição usa dados de reclamações e sugestões para melhorar o } \\
\text { serviço? }\end{array}$ & Sim/Não \\
\hline Q16 & Existe uma medida de custo total para o usuário? & \\
\hline & &
\end{tabular}




\begin{tabular}{|c|c|c|}
\hline Q17 & $\begin{array}{l}\text { Existe uma ferramenta para o usuário avaliar sua satisfação com o } \\
\text { serviço? }\end{array}$ & Sim/Não \\
\hline Q18 & Os usuários estão envolvidos nos processos de melhoria de serviço? & Sim/Não \\
\hline Q19 & $\begin{array}{l}\text { A instituição é proativa em fornecer informações de interesse dos } \\
\text { usuários sobre os serviços }\end{array}$ & Likert 4 \\
\hline Q20 & $\begin{array}{l}\text { Os requisitos documentais para a prestação do serviço podem ser } \\
\text { reduzidos. }\end{array}$ & Likert 4 \\
\hline Q21 & $\begin{array}{l}\text { A instituição possui canais de divulgação de informações sobre } \\
\text { desempenho de serviços e ações de melhoria. }\end{array}$ & Likert 4 \\
\hline Q22 & O serviço está incluído no plano estratégico da instituição? & Sim/Não \\
\hline Q23 & $\begin{array}{l}\text { A instituição segue um modelo de gestão da qualidade definido para a } \\
\text { prestação do serviço? }\end{array}$ & $\mathrm{Sim} / \mathrm{Não}$ \\
\hline Q24 & $\begin{array}{l}\text { A instituição possui um processo regular de avaliação da qualidade do } \\
\text { serviço prestado? }\end{array}$ & Sim/Não \\
\hline Q25 & Existem pessoas dedicadas especificamente à avaliação da qualidade? & $\mathrm{Sim} / \mathrm{Não}$ \\
\hline Q26 & $\begin{array}{l}\text { A alta gerência avalia a coerência entre a missão da instituição e os } \\
\text { serviços que ela oferece. }\end{array}$ & Likert 4 \\
\hline Q27 & $\begin{array}{l}\text { A alta gerência monitora os padrões de qualidade dos serviços } \\
\text { prestados. }\end{array}$ & Likert 4 \\
\hline Q28 & A alta gerência patrocina a divulgação dos serviços no site dos serviços. & Likert 4 \\
\hline Q29 & $\begin{array}{l}\text { A alta gerência promove melhorias nos processos de gerenciamento, } \\
\text { com o objetivo de reduzir os requisitos para os usuários do serviço. }\end{array}$ & Likert 4 \\
\hline Q30 & $\begin{array}{l}\text { Existem alianças, parcerias, iniciativas de colaboração e / ou integração } \\
\text { com outras instituições públicas que visam melhorar o serviço? }\end{array}$ & Sim/Não \\
\hline Q31 & $\begin{array}{l}\text { Existem ações de treinamento associadas ao serviço analisado } \\
\text { (considere apenas o treinamento vinculado ao serviço analisado, não } \\
\text { a instituição como um todo)? }\end{array}$ & Sim/Não \\
\hline Q32 & $\begin{array}{l}\text { Os incentivos oferecidos aos funcionários por seu desempenho estão } \\
\text { associados à melhoria da qualidade do serviço? }\end{array}$ & Sim/Não \\
\hline Q33 & $\begin{array}{l}\text { A instituição promove a transferência de conhecimento entre os } \\
\text { funcionários. }\end{array}$ & Likert 4 \\
\hline Q34 & Existe uma política de treinamento para todos os funcionários. & Likert 4 \\
\hline Q35 & O número atual de funcionários é adequado para prestar o serviço. & Likert 4 \\
\hline Q36 & $\begin{array}{l}\text { Os funcionários são treinados para prestar o serviço assim que iniciam } \\
\text { suas atividades. }\end{array}$ & Likert 4 \\
\hline \multirow[t]{2}{*}{ Q37 } & $\begin{array}{l}\text { Os funcionários são selecionados de acordo com as habilidades } \\
\text { necessárias para prestar o serviço. }\end{array}$ & Likert 4 \\
\hline & Qual das seguintes ações pode ser executada pelo usuário pela Internet? & \\
\hline Q38 & $\begin{array}{l}\text { Acessar informações sobre os documentos necessários para solicitar o } \\
\text { serviço }\end{array}$ & Sim/Não \\
\hline Q39 & Acessar informações passo a passo sobre como solicitar o serviço & Sim/Não \\
\hline Q40 & Agendar Serviço & Sim/Não \\
\hline Q41 & Consulta Agendamento & Sim/Não \\
\hline Q42 & Requisitar o serviço & Sim/Não \\
\hline Q43 & Acompanhar o serviço & Sim/Não \\
\hline Q44 & Registrar sugestão ou reclamação & Sim/Não \\
\hline Q45 & Receber resposta de uma solicitação, requisição ou reclamação & Sim/Não \\
\hline
\end{tabular}




\begin{tabular}{|l|l|l|}
\hline Q46 & Assinar documento & Sim/Não \\
\hline Q47 & Realizar Pagamento & Sim/Não \\
\hline Q48 & Os sistemas são confiáveis em termos de disponibilidade & Likert 4 \\
\hline Q49 & Os sistemas facilitam o gerenciamento diário do serviço & Likert 4 \\
\hline Q50 & Os sistemas fornecem dados e informações confiáveis & Likert 4 \\
\hline Q51 & Os sistemas existentes garantem suporte completo ao serviço fornecido & Likert 4 \\
\hline Q52 & Os sistemas fornecem os dados dos serviços no formato aberto & Likert 4 \\
\hline Q53 & Os sistemas atendem aos requisitos de acessibilidade & Likert 4 \\
\hline Q54 & $\begin{array}{l}\text { As estratégias regulares de divulgação do serviço para o usuário são } \\
\text { efetivas. }\end{array}$ & Likert 4 \\
\hline
\end{tabular}

\section{Materiais e Métodos}

O objetivo deste trabalho é desenvolver dois modelos preditivos do tempo de espera dos serviços públicos brasileiros: um modelo para serviços oferecidos a pessoas físicas e outro a pessoas jurídicas. Essa divisão se deve ao interesse em diferenciar o comportamento de cada tipo de serviço, uma vez que a demanda entre os dois tipos são diferentes, assim como os custos envolvidos, tanto da parte do usuário quanto da Administração Pública.

O instrumento de avaliação (detalhado na seção anterior) contou com a participação de 52 instituições federais. Ao todo 211 gestores responderam ao questionário, sendo que 78 gestores coordenavam mais de um serviço.

\subsection{Caracterização da base de dados}

Do total de respostas obtidas através do questionário aplicado aos gestores, foram analisados 98 serviços públicos destinados a pessoas físicas. A Figura 1 apresenta a distribuição desses serviços segundo seu tempo de espera. Nota-se que, para serviços ofertados a pessoas físicas, existe uma amostra maior para serviços com tempo de espera baixo (até 30 dias).

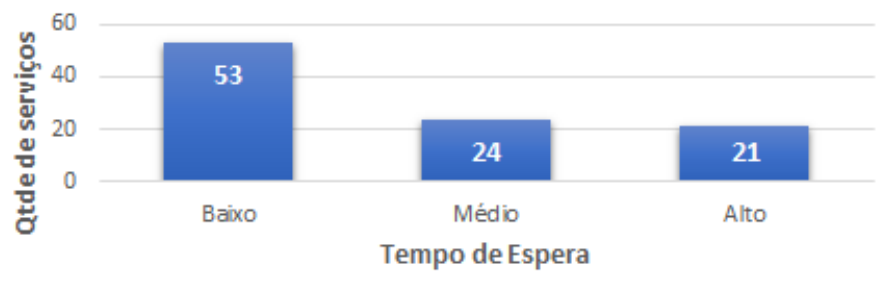

\section{Figura 1. Distribuição do tempo de espera dos serviços da base de dados que são ofertados a pessoas físicas.}

De fato, os serviços que envolvem pessoas físicas possuem um tempo de espera menor quando comparados aos serviços direcionados a pessoas jurídicas. Isso se deve ao fato de que grande parte dos serviços da Administração Pública está relacionada ao atendimento presencial do usuário na instituição, tais como: obtenção de licenças, documentos, certificados, etc.

Já para os serviços direcionados a pessoas jurídicas, foram obtidos dados referentes a 109 dos serviços públicos. A Figura 2 apresenta a distribuição desses dados conforme o nível do tempo de espera: 47 serviços com nível baixo, 33 com nível médio e 29 com nível alto. 


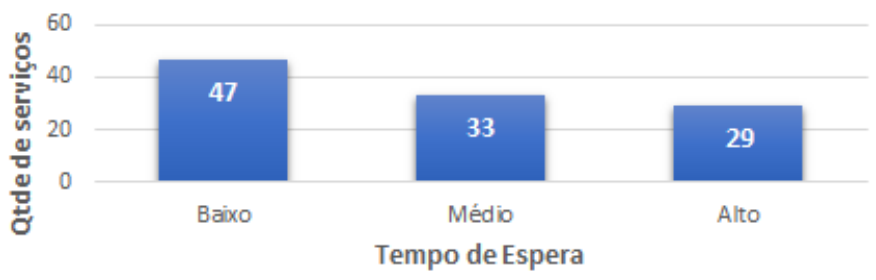

Figura 2. Distribuição de serviços entregues a pessoas jurídicas segundo seu tempo de espera.

\subsection{Algoritmos de Aprendizado de Máquinas}

Para a realização dos testes experimentais foi utilizado o $\mathrm{Weka}^{1}$, que é um aplicativo voltado à mineração de dados que possui uma interface gráfica que permite carregar um conjunto de dados, mostrar estatísticas, escolher e configurar o algoritmo de aprendizado de máquina a ser utilizado, e, obviamente, executar o método escolhido.

\subsubsection{Classificação e Validação}

Para fins de comparação foram selecionados sete algoritmos de classificação com abordagens diferentes:

- SVM

- J48: implementação do algoritmo C4.5 baseado em Árvore de Decisão

- Naive Bayes

- KNN : (k-Vizinhos mais Próximos). Nos testes experimentais foi utilizado $\mathrm{K}=5$;

- Random Forest

- Multilayer Perceptron: rede neural com apenas 1 camada cuja quantidade de neurônios é dada pela quantidade de entradas

- Regressão Logística Simples

Todas essas técnicas estão implementadas pela plataforma do Weka. Neste trabalho, cada algoritmo classificador foi testado com validação cruzada com 10 partições diferentes através do software Weka v3.8.3.

A técnica de cross-validation é usada para medir como os resultados de uma análise estatística vão ser generalizados para um conjunto de dados independente. Ela é principalmente usada em configurações onde o objetivo é a predição, e alguém deseja estimar o quão correto um modelo preditivo irá ser executado na prática. Uma rodada do cross-validation envolve o particionamento de uma amostra de dados em subconjuntos complementares, executando a análise de um subconjunto (chamado de conjunto de treinamento), e validando a análise em outro subconjunto (chamado de conjunto de validação ou teste). Para reduzir a variabilidade, múltiplas rodadas do cross-validation são executadas usando diferentes partições, e os resultados de validação são a média das rodadas.

Para avaliação do desempenho das técnicas de classificação foram analisadas três métricas:

\footnotetext{
${ }^{1} \mathrm{http}: / /$ www.cs.waikato.ac.nz/ml/weka/
} 
- Acurácia: indica a quantidade de dados classificados corretamente pelo classificador

- F1-Score: $2 *$ Precisao*Revocacao

- AUC: área abaixo da curva ROC

\subsubsection{Seleção de Atributos}

A seleção de atributos é uma técnica de mineração de dados aplicada para reduzir a dimensionalidade dos dados, facilitando a aplicação de algoritmos de mineração. A redução da dimensionalidade produz uma representação mais compacta, mais facilmente interpretável do conceito alvo, focalizando a atenção sobre as variáveis mais relevantes [Witten and Frank 2009].

A seleção de atributos é um recurso importante que afeta o desempenho de um modelo de predição. Dados irrelevantes podem diminuir a precisão dos modelos, por isso é importante, neste trabalho, identificar quais as variáveis do questionário aplicado aos gestores possuem uma maior correlação com o tempo de espera do serviço.

A correlação entre variáveis é mais formalmente referida como coeficiente de correlação de Pearson nas estatísticas. É possível calcular a correlação entre cada atributo e a variável de saída e selecionar apenas os atributos que possuem uma correlação positiva ou negativa de moderada a alta (perto de -1 ou 1) e eliminar esses atributos com uma correlação baixa (valor próximo a zero ).

Neste trabalho, o algoritmo utilizado para realizar a seleção de características foi o CFS (Correlation Based Feature Selection) [Hall 1998]. Em suma, o objetivo do CFS é detectar um conjunto de variáveis não correlacionadas entre si, mas que apresentam alta correlação com a variável preditiva. No Weka, a implementação da técnica CFS está referenciada como CfsSubsetEval. Foi utilizado o método de busca do tipo "BestFirst".

\section{Resultados e Discussões}

A acurácia de cada classificador foi avaliada em duas fases: na primeira sem a seleção de atributos e outra considerando apenas as variáveis selecionadas pelo algoritmo CFS. Os resultados são apresentados a seguir.

\subsection{Resultados Preliminares: Fase 1}

A Tabela 2 consolida alguns valores de acurácia gerados no aprendizado da relação entre os serviços públicos entregues a pessoas físicas e seu tempo de espera, juntamente com seus algoritmos geradores. Já a Tabela 3 apresenta os resultados dos algoritmos classificadores para serviços públicos entregues a pessoas jurídicas. A partir desses resultados preliminares, foi realizado um novo teste para avaliar se a técnica de seleção de atributos seria realmente eficaz na melhoria do poder preditivo dos classificadores. 
Tabela 2. Resultados de alguns classificadores para serviços públicos entregues a pessoas físicas

\begin{tabular}{|l|l|l|l|}
\hline Classificador & Acurácia & F-1 & AUC \\
\hline Naive Bayes & $55,1 \%$ & 0,552 & 0,737 \\
\hline J48 & $52,04 \%$ & 0,522 & 0,617 \\
\hline Random Forest & $61,2 \%$ & 0,533 & 0,812 \\
\hline SVM & $64,29 \%$ & 0,638 & 0,716 \\
\hline MultiLayer Perceptron & $60,20 \%$ & 0,601 & 0,769 \\
\hline Regressão Logística & $66,33 \%$ & 0,67 & 0,71 \\
\hline KNN & $57,14 \%$ & 0,502 & 0,683 \\
\hline
\end{tabular}

Tabela 3. Resultados de alguns classificadores para serviços públicos ofertados a pessoas jurídicas

\begin{tabular}{|l|l|l|l|}
\hline Classificador & Acurácia & F-1 & AUC \\
\hline Naive Bayes & $50,5 \%$ & 0,506 & 0,678 \\
\hline J48 & $53,21 \%$ & 0,524 & 0,621 \\
\hline Random Forest & $54,1 \%$ & 0,523 & 0,723 \\
\hline SVM & $47,71 \%$ & 0,475 & 0,662 \\
\hline MultiLayer Perceptron & $49,54 \%$ & 0,498 & 0,666 \\
\hline Regressão Logística & $61,47 \%$ & 0,61 & 0,714 \\
\hline KNN & $42,20 \%$ & 0,41 & 0,656 \\
\hline
\end{tabular}

\subsection{Resultados Após Seleção de Atributos: Fase 2}

Frente aos resultados preliminares, foi realizada uma seleção de atributos (questões), sobre o questionário aplicado, a fim de selecionar um conjunto reduzido de questões correlacionadas à variável preditora (tempo de espera). A Tabela 4 apresenta as variáveis selecionadas após a aplicação da técnica de seleção de atributos, discutida na seção anterior, para serviços oferecidos à pessoas físicas e a Tabela 5 para serviços direcionados à pessoas jurídicas.

Tabela 4. Variáveis selecionadas para serviços públicos direcionados a pessoas físicas

\begin{tabular}{|l|l|}
\hline Q3 & Quantas áreas da instituição estão envolvidas na prestação do serviço? \\
\hline Q4 & Quantas vezes o usuário interage com a instituição até a entrega do serviço? \\
\hline Q5 & Quantos documentos o usuário necessita apresentar para a realização do serviço? \\
\hline Q38 & Ações realizadas pela internet: informações sobre documentos \\
\hline Q44 & Ações realizadas pela internet: registrar sugestão/reclamação \\
\hline Q16 & Há mensuração do custo total para o usuário? \\
\hline Q24 & $\begin{array}{l}\text { A instituição possui processo regular de avaliação da qualidade do serviço } \\
\text { prestado? }\end{array}$ \\
\hline
\end{tabular}


Tabela 5. Variáveis selecionadas para serviços públicos ofertados a pessoas jurídicas

\begin{tabular}{|l|l|}
\hline Q4 & Quantas vezes o usuário interage com a instituição até a entrega do serviço? \\
\hline Q5 & Quantos documentos o usuário necessita apresentar para a realização do serviço? \\
\hline Q38 & Ações realizadas pela internet: informações sobre documentos \\
\hline Q16 & Há mensuração do custo total para o usuário? \\
\hline Q25 & Há pessoas especificamente dedicadas à avaliação da qualidade? \\
\hline
\end{tabular}

Após essa seleção de atributos (questões), os algoritmos de classificação foram novamente testados para cada tipo de serviço com seu respectivo conjunto de variáveis selecionadas. A Tabela 6 apresenta a acurácia dos classificadores para aqueles serviços ofertados exclusivamente à pessoas físicas. Observa-se que a maior acurácia, de 76,8\%, é gerada pelo algoritmo Naïve-Bayes. Nota-se, ainda, que este algoritmo também apresentada as melhores medidas de F1-Score e ROC, tornando-o o classificador mais adequado para predição do tempo de espera considerando os serviços voltados a pessoas físicas.

Tabela 6. Acurácia dos classificadores para serviços públicos direcionados a pessoas físicas após a seleção de variáveis

\begin{tabular}{|l|c|c|c|}
\hline Classificador & Acurácia & F-1 & AUC \\
\hline Naive Bayes & $76,8 \%$ & 0,752 & 0,882 \\
\hline J48 & $60,20 \%$ & 0,559 & 0,621 \\
\hline Random Forest & $66,3 \%$ & 0,634 & 0,855 \\
\hline SVM & $72,45 \%$ & 0,717 & 0,828 \\
\hline MultiLayer Perceptron & $72,45 \%$ & 0,721 & 0,858 \\
\hline Regressão Logística & $71,43 \%$ & 0,704 & 0,852 \\
\hline KNN & $66,33 \%$ & 0,617 & 0,788 \\
\hline
\end{tabular}

Já a Tabela 7 apresenta a acurácia dos classificadores para os serviços ofertados a pessoas jurídicas. A maior acurácia, de $69,72 \%$, é obtida pelo algoritmo J48, que também apresenta os melhores valores F1 e ROC, tornando-o o modelo adequado para este tipo de serviço.

Tabela 7. Acurácia dos classificadores para serviços públicos entregues a pessoas jurídicas após a seleção de variáveis.

\begin{tabular}{|l|c|c|c|}
\hline Classificador & Acurácia & F-1 & AUC \\
\hline Naive Bayes & $64,2 \%$ & 0,641 & 0,611 \\
\hline J48 & $69,72 \%$ & 0,676 & 0,795 \\
\hline Random Forest & $65,1 \%$ & 0,645 & 0,789 \\
\hline SVM & $65,14 \%$ & 0,65 & 0,754 \\
\hline MultiLayer Perceptron & $61,47 \%$ & 0,613 & 0,773 \\
\hline Regressão Logística & $65,14 \%$ & 0,651 & 0,772 \\
\hline KNN & $59,63 \%$ & 0,586 & 0,751 \\
\hline
\end{tabular}

A Tabela 8 apresenta a matriz de confusão do algoritmo Naïve-Bayes para predição do tempo de espera de serviços públicos ofertados a pessoas físicas. Matrizes 
de confusão são representações sumarizadas que permitem visualizar a quantidade de predições corretas e incorretas por classe. Quanto mais próximo de $100 \%$ melhor é a acurácia (taxa de acerto). Por exemplo, nota-se, pela matriz de confusão da Tabela 8, que o pior desempenho da técnica foi na classificação de serviços com tempo de espera médio, visto que o menor valor de acurácia para essa classe foi de apenas $46 \%$.

Tabela 8. Matriz de confusão do algoritmo Naïve-Bayes para predição do tempo de espera de serviços públicos ofertados a pessoas físicas

\begin{tabular}{|l|l|l|l|}
\hline & baixo & médio & alto \\
\hline baixo & $92 \%$ & $4 \%$ & $4 \%$ \\
\hline médio & $42 \%$ & $46 \%$ & $13 \%$ \\
\hline alto & $24 \%$ & $5 \%$ & $71 \%$ \\
\hline
\end{tabular}

Já para os serviços ofertados a pessoas jurídicas, a Tabela 9 mostra a matriz de confusão do algoritmo J48. Nota-se que os maiores valores encontram-se na diagonal do matriz, logo, o algoritmo obteve êxito na classificação em todos os níveis de espera. Quanto mais próximo de $100 \%$, maior é a taxa de acerto.

Tabela 9. Matriz de confusão do algoritmo J48 para predição do tempo de espera de serviços públicos ofertados a pessoas jurídicas

\begin{tabular}{|l|l|l|l|}
\hline & baixo & médio & alto \\
\hline baixo & $89 \%$ & $4 \%$ & $6 \%$ \\
\hline médio & $36 \%$ & $45 \%$ & $18 \%$ \\
\hline alto & $17 \%$ & $10 \%$ & $73 \%$ \\
\hline
\end{tabular}

\subsection{Discussão}

Os resultados mostram uma forte correlação entre a quantidade de documentos necessários para obtenção do serviço e o tempo de espera, tanto para aqueles direcionados a pessoas físicas quanto para pessoas jurídicas. De fato, observando a base de dados, notase que os serviços que demandam 0,1 ou 2 documentos estão concentrados no nível mais baixo do tempo de espera, enquanto aqueles que demandam mais documentos possuem um tempo de espera mais elevado.

A quantidade de documentos que o usuário deve fornecer ao serviço, está relacionada a outro aspecto importante que afeta negativamente o tempo de espera que é o acesso às informações sobre os documentos do serviço pela internet. Isso significa que, garantir que o usuário esteja bem informado sobre os documentos do serviço aumenta a chance que ele possua todos os documentos necessários em sua primeira tentativa de se obter o serviço. Nesse sentido, melhorar as estratégias de informação documental do serviço pode evitar que o usuário deixe de fornecer dados relevantes para a obtenção do serviço.

A quantidade de interações do usuário também se mostrou uma variável relevante para prever o tempo de espera dos serviços. Novamente, quanto menor a quantidade de interações, menor o tempo de espera do serviço. Portanto, é importante que os gestores consolidem a entrega de seus serviços de maneira a reduzir a quantidade de ocasiões em 
que o usuário deve interagir com a instituição e, assim, minimizar esforços desnecessários que possam gerar a necessidade por novas interações do usuário com a instituição pública.

Finalmente, os resultados destacam também para a presença de um processo regular de avaliação da qualidade ou de pessoas dedicadas a essa avaliação. De maneira geral, serviços em que tais aspectos estão presentes possuem um tempo de espera menor. Desse modo, avaliar a qualidade e a gestão da qualidade mostra-se essencial para aprimorar o tempo de espera dos serviços e assim impulsionar sua qualidade.

\section{Conclusão}

Este artigo apresentou os resultados de um estudo acerca dos dados da pesquisa de gestão da qualidade realizada pelo Governo Federal brasileiro nas instituições públicas. Com o auxílio das técnicas de aprendizado de máquina foram construídos dois modelos preditivos do tempo de espera de serviços públicos ofertados a pessoas físicas e jurídicas com base em aspectos da sua gestão da qualidade. O tempo de espera analisado neste trabalho se refere ao período (em dias) da duração desde a solicitação do serviço até a efetiva entrega ao usuário solicitante.

Ao se aplicar um método de seleção de atributos, foi possível observar algumas variáveis de gestão altamente correlacionadas com o tempo de espera dos serviços. Alguns aspectos organizacionais foram relevantes na geração dos dois modelos de predição, como: a quantidade de documentos que o usuário deve fornecer, e a quantidade de vezes que o usuário interage com a instituição provedora do serviço. Tais variáveis contribuem fortemente com atrasos na entrega do serviço e, consequentemente, em um tempo de espera maior.

Nos experimentos realizados, o algoritmo Naïve-Bayes apresentou os melhores resultados de acurácia para predição do tempo de espera em serviços direcionados a pessoas físicas. Enquanto o algoritmo J48, que é baseado em árvore de decisão, apresentou a melhor acurácia para serviços públicos ofertados a pessoas jurídicas.

Em suma, os resultados desse estudo indicam a importância da gestão da qualidade para a melhoria dos serviços públicos. A partir dos modelos construídos, gestores de serviços podem compreender melhor a relação entre aspectos dos serviços e o tempo de espera. Com isso, os gestores poderão elaborar estratégias adequadas para a minimização do tempo de espera, visto que é uma característica importante para a qualidade dos serviços e satisfação dos usuários, sobretudo atualmente com a tendência para estratégias orientadas ao usuário.

Como trabalho futuro, está sendo elaborada uma nova versão do questionário e pretende-se aplica-lo novamente com os gestores de serviços púbicos. Nesta nova versão, alguns novos aspectos estão sendo detalhados, por exemplo, o custo envolvido tanto para o usuário (consumidor do serviço) quanto o custo para o Governo, bem como identificar como os usos de SI na gestão dos serviços podem afetar a qualidade percebida pelo usuário.

\section{Referências}

Alanezi, M. A., Mahmood, A. K., and Basri, S. (2011). Conceptual model for measuring e-government service quality. In 2011 IEEE Conference on Open Systems, pages 411416. 
Arias, M. I. and Maçada, A. C. G. (2018). Digital government for e-government service quality: A literature review. In Proceedings of the 11th International Conference on Theory and Practice of Electronic Governance, ICEGOV '18, pages 7-17, New York, NY, USA. ACM.

Bielen, F. and Demoulin, N. (2007). Waiting time influence on the satisfactionloyalty relationship in services. Managing Service Quality: An International Journal, 17(2):174-193.

Hall, M. A. (1998). Correlation-based Feature Subset Selection for Machine Learning. $\mathrm{PhD}$ thesis, University of Waikato, Hamilton, New Zealand.

Manocha, P., Som, S., and Chanana, L. (2018). Technological Trends, Impact and Analysis of Social Media Quality Parameters on e-Governance Applications. In 2018 International Conference on System Modeling \& Advancement in Research Trends (SMART), pages 280-285, Moradabad, India. IEEE.

Pareja, A., Fernandez, C., Blanco, B., Theobald, K., and Martinez, A. (2016). Simplificando vidas: qualidade e satisfação com os serviços públicos. Banco Interamericano de Desenvolvimento (BID).

Soares, V., Yukari, G., Menezes, V., Gomes, M., Pedrosa, G., Silva, W., and Figueiredo, R. (2019). Evaluating government services based on user perspective. In Proceedings of the 20th Annual International Conference on Digital Government Research, dg.o 2019, pages 425-432, New York, NY, USA. ACM.

Witten, I. H. and Frank, E. (2009). Data Mining: Practical machine learning tools and techniques with java implementations. Massachusetts: Morgan Kaufmann. 